\title{
OPEN Effects of brief sodium fluoride treatments on the growth of early and mature cariogenic biofilms
}

\author{
Ye Han
}

Although fluoride has been widely used as a preventive agent for dental caries, the effects of fluoride on the activities of biofilms in different stages of cariogenic biofilm formation are less studied. This study was designed to investigate the antibiofilm activity of sodium fluoride during the early and mature stages of Streptococcus mutans (S. mutans) biofilm formation. S. mutans biofilms were formed on saliva-coated hydroxyapatite disks. In the early ( $0-46 \mathrm{~h}$ ) and mature (46-94 h) biofilm stages, the biofilms were treated with different concentrations of fluoride $(250,500,1000,2000$ ppm; 5 times in total, $1 \mathrm{~min} /$ treatment). Acidogenicity, dry weight, colony-forming units (CFUs), water-soluble/ insoluble extracellular polysaccharides (EPSs), and intracellular polysaccharides were analysed, and confocal laser scanning microscopy images were obtained of the two stages of biofilms to determine antibiofilm activities of fluoride at varying concentrations during the formation of early and mature biofilms. In the early stages of cariogenic biofilm formation, test groups with all fluoride concentrations significantly inhibited the growth of $S$. mutans biofilms. The antibiofilm and antiEPS formation activities of the brief fluoride treatments increased with a concentration-dependent pattern. At the mature biofilm stage, only the $2000 \mathrm{ppm}$ fluoride treatment group significantly inhibited biofilm accumulation, activity, and intracellular/extracellular polysaccharide content compared with those of the control and other fluoride treatment groups. The antimicrobial effect of fluoride treatment on the growth of $S$. mutans biofilms was linked with the stage of cariogenic biofilm formation. The inhibition of $S$. mutans biofilm growth by fluoride treatment was easier in the early formation stage than in the mature stage. Fluoride treatment in the early stage of cariogenic biofilm formation may be an effective approach to controlling cariogenic biofilm development and preventing dental caries.

Dental caries is an oral disease associated with dental biofilms ${ }^{1}$. The increased levels of acidogenic and aciduric bacteria in microorganism are of great significance in the pathogenesis of dental caries ${ }^{1}$. Through glycolysis, acidogenic bacteria reduce the $\mathrm{pH}$ of dental biofilms developed on the surfaces of teeth exposed to dietary sugar $^{1}$. The low $\mathrm{pH}$ environment further accelerates the growth of acidic and acidogenic bacteria, including mutans streptococci, other acidogenic streptococci, lactobacilli, and bifidobacteria ${ }^{2}$. The compositions of cariogenic biofilm microflora are complex ${ }^{3}$. Although there may be other acidogenic and aciduric bacteria ${ }^{4}, S$. mutans is considered one of the important aetiological agents for the development of dental caries ${ }^{5,6}$. S. mutans can produce glucosyltransferases and synthesize extracellular polysaccharides with sucrose as a substrate. EPSs contribute to bacterial adhesion on the tooth surface, colonization, and formation of plaque biofilms, which are important cariogenic factors of $S$. mutans ${ }^{7}$. In addition, acid production through carbohydrates and acid tolerance at low $\mathrm{pH}$ are also the major cariogenic factors of $S$. mutans that result in the loss of local hard tissues and initiation of the cariogenic process ${ }^{7}$. Decreases in $S$. mutans adhesion to tooth surfaces, acid production, and EPS formation by $S$. mutans biofilms may reduce the incidence of dental caries.

Fluoride is a widely used preventive agent for dental caries. Fluoride products, such as fluoride water, mouthwash, ordinary toothpaste, and prescription toothpaste, play an important role in preventing dental caries ${ }^{8}$. Reducing enamel solubility and promoting its remineralization are considered the underlying mechanisms of fluoride action against dental caries ${ }^{9,10}$. Previous findings have shown that fluoride inhibits the adhesion of $S$. mutans to the surface of hydroxyapatite ${ }^{11}$ and also inhibits acid production, acid tolerance, and glucosyltransferase production of $S$. mutans biofilms $s^{12-14}$. In addition, the effect of fluoride on cariogenic biofilm virulence has been identified. Even one 1-min fluoride treatment with a minimum concentration of $300 \mathrm{ppm}$ can affect 

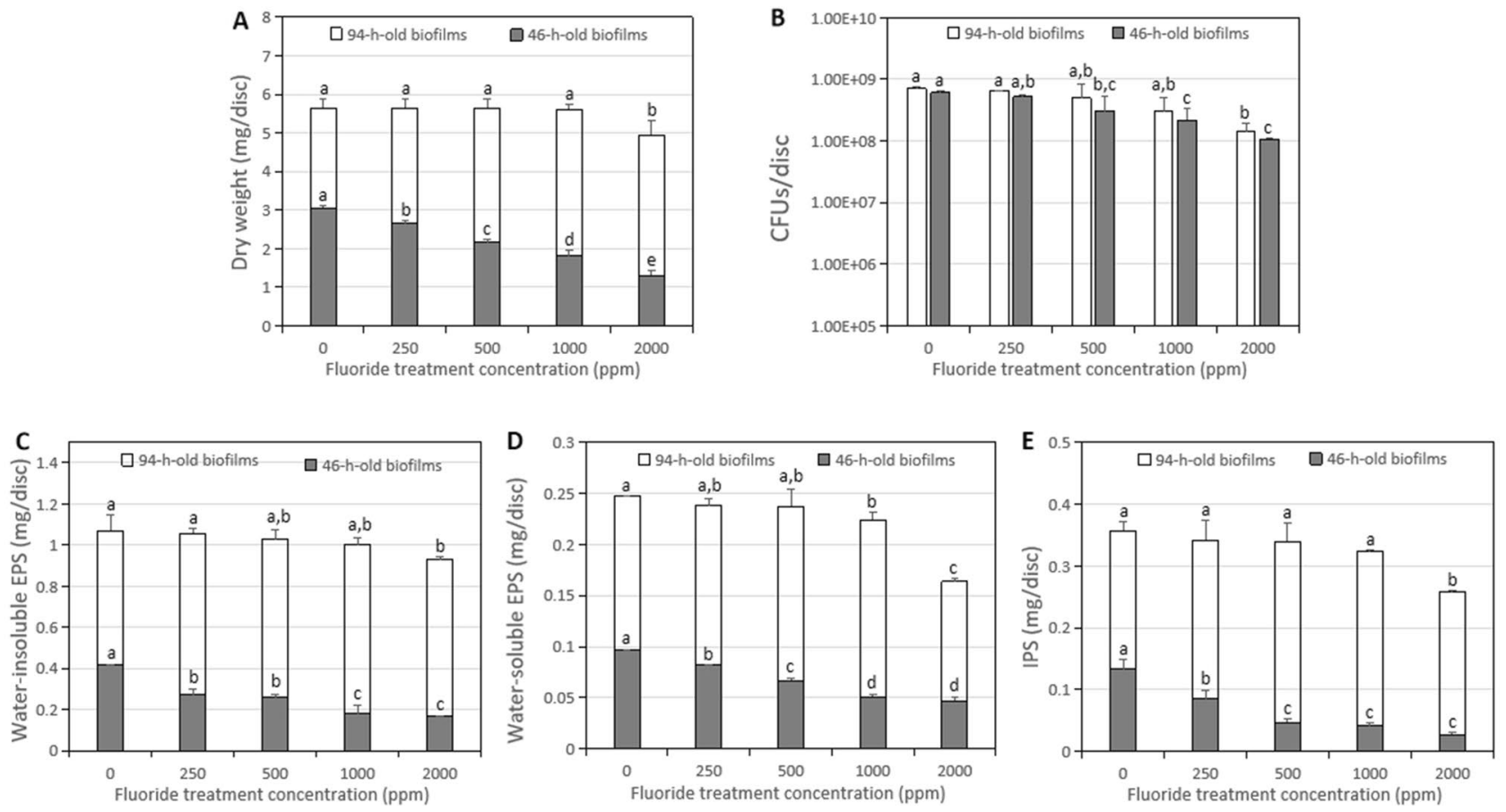

Figure 1. Change in the microbiological and biochemical composition of the 46/94-h-old S. mutans biofilms treated with different concentrations of fluoride. (A) Dry weight. (B) CFUs. (C) Water-insoluble EPSs. (D) Water-soluble EPSs. (E) Intracellular polysaccharides (IPS). Data represent mean \pm standard deviation. In the 46-h-old $S$. mutans biofilms, (A-E), $p<0.05$. In the 94-h-old $S$. mutans biofilms, figure (A-C), $p>0.05$; (D,E), $p<0.05 .{ }^{*} p<0.05$ : significantly different from each other. $p>0.05$ : values followed by the same superscript are not significantly different from each other.

the cariogenic characteristics of $S$. mutans biofilms ${ }^{15}$, although it cannot sustain antiviral activity because of the recovery of $S$. mutans from fluoride shock over time ${ }^{16}$. Periodic 1-min fluoride treatments, however, reduce the dry weight and acid production of $S$. mutans biofilms in a concentration-dependent manner ${ }^{17}$. A growing number of studies have focused on the mechanism by which fluoride inhibits the cariogenic biofilm growth of S. mutans. Nevertheless, the relationship between the anticariogenic biofilm activity of fluoride and the growth stages of biofilms as well as the effect of brief fluoride treatments on the virulence of early and mature cariogenic biofilms remain largely unclear. It is necessary to validate the hypothesis that brief fluoride treatments yield anticariogenic biofilm activity, mainly through the early stage of biofilm formation. Therefore, this study is designed to demonstrate the effects of fluoride on the development of $S$. mutants biofilms that depend on the biofilm formation stage. In brief, $S$. mutans biofilms were treated with different concentrations of fluoride $(0,250$, $500,1000,2000 \mathrm{ppm}$ ) in the following two stages: early biofilm formation stage at $0-46 \mathrm{~h}$ and mature biofilm stage at 46-94 h. The effects of varying concentrations of fluoride on the development of $S$. mutans biofilms in the two stages were compared.

\section{Result}

Effects of brief fluoride treatments during early $S$. mutans biofilms formation. The microbiological and biochemical analyses in the early S. mutans biofilms formation. As shown in Fig. 1, brief fluoride treatments with all tested concentrations significantly reduced the dry weight accumulations, bacterial activities, and polysaccharides formation of 46 -h-old $S$. mutans biofilms in a concentration-dependent manner, and the differences between each treatment group and the control group were statistically significant (Fig. 1A-E; $p<0.05)$. The lowest values were found for the $2000 \mathrm{ppm}$ fluoride treatment group.

Relationship between fluoride concentration and acid production of early $S$. mutans biofilms. Figure $2 \mathrm{~A}$ showed the $\mathrm{pH}$ changes of the old culture medium, it was found that all tested concentrations of fluoride treatment groups inhibited the acid-producing capacity of the biofilm in the early formation stage of the biofilm $(p<0.05)$. The concentration of acid in the old medium decreased in a concentration-dependent manner, and the highest inhibition ability was shown for the $2000 \mathrm{ppm}$ fluoride treatment group. The glycolytic $\mathrm{pH}$ drop assay was used to determine the effect of brief fluoride treatment on the acid production and acid tolerance of 46-h-old S. mutans biofilms. As shown in Fig. 3A,B, brief fluoride treatments inhibited acid production in the early stage of cariogenic biofilms. The acidification activities of biofilms were inhibited in all fluoride treatment groups, and the $2000 \mathrm{ppm}$ fluoride treatment group showed the lowest initial rate and total acid production (Fig. 3B). Additionally, the 2000 ppm fluoride treatment group showed reduced acid resistance of cariogenic 

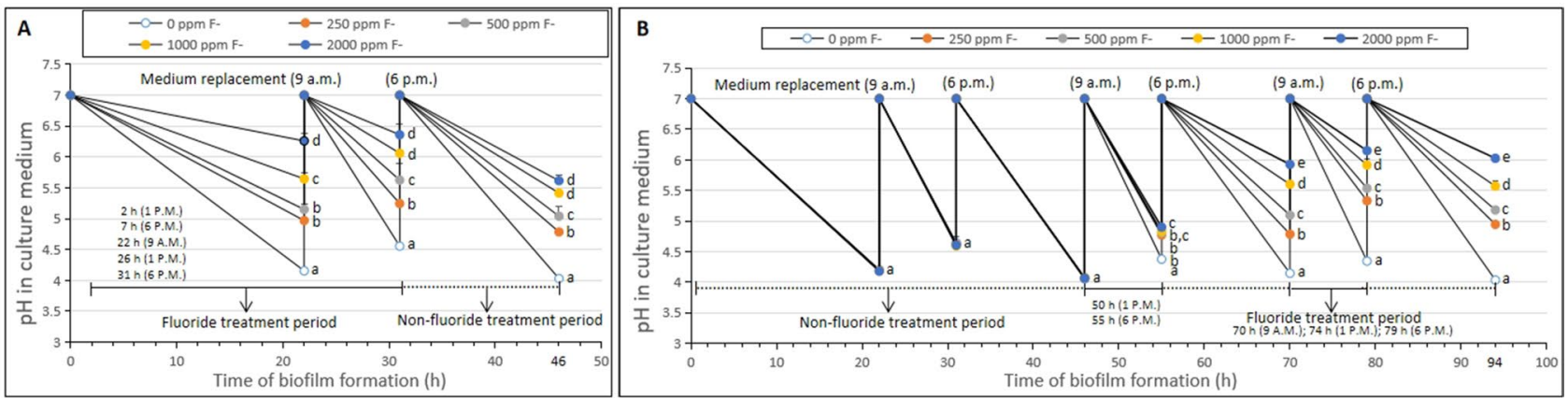

Figure 2. Change in the $\mathrm{pH}$ values of old culture medium treated with different concentrations of fluoride during 46/94-h-old $S$. mutans biofilms formation. (A) 46-h-old $S$. mutans biofilms $(p<0.05)$. (B) 94-h-old $S$. mutans biofilms $(22,31,46 \mathrm{~h}, p>0.05 ; 55,70,79,94 \mathrm{~h}, p<0.05)$. Data represent mean \pm standard deviation. ${ }^{*} p<0.05$ : significantly different from each other. $p>0.05$ : values followed by the same superscript are not significantly different from each other.
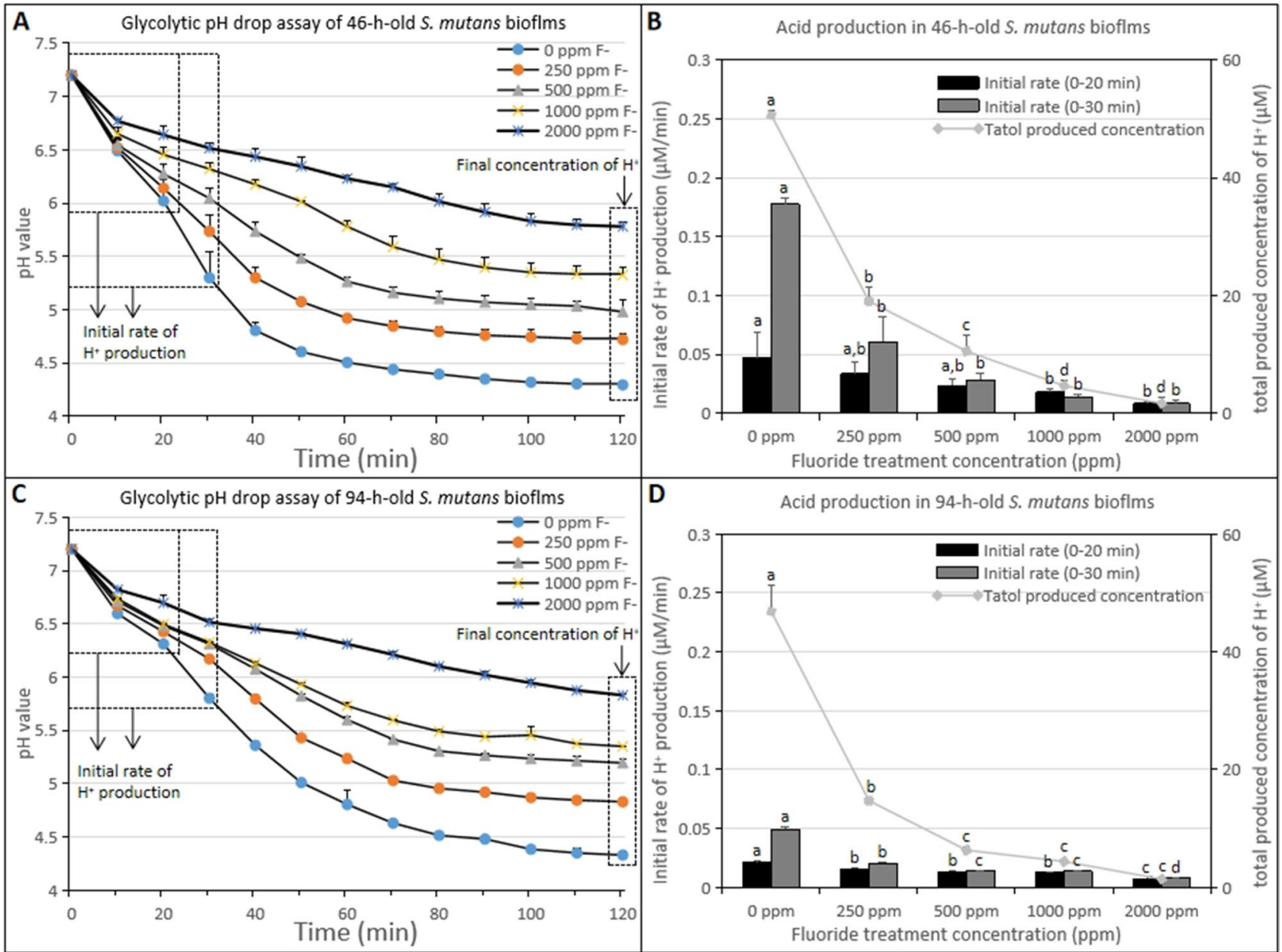

Figure 3. The effect of different concentrations of fluoride treatment on the acidogenicity of 46/94-h-old $S$. mutans biofilms cells. Changes in the initial rate of $\mathrm{H}^{+}$production $(0-20 \mathrm{~min} ; 0-30 \mathrm{~min})$ and total produced concentration of $\mathrm{H}^{+}$(120 min) in 46/94-h-old $S$. mutans biofilms, calculated from biofilm glycolytic $\mathrm{pH}$ drop assay data. (A) Change in acid production of 46 -h-old $S$. mutans biofilms $(p<0.05)$. (B) Initial rate of $\mathrm{H}^{+}$ production (0-20 min; 0-30 min) and total produced concentration of $\mathrm{H}^{+}(120 \mathrm{~min})$ in 46-h-old S. mutans biofilms $(p<0.05)$. (C) Change in acid production of 94-h-old $S$. mutans biofilms. (D) Initial rate of $\mathrm{H}^{+}$ production (0-20 $\mathrm{min} ; 0-30 \mathrm{~min})$ and total produced concentration of $\mathrm{H}^{+}(120 \mathrm{~min})$ in 94-h-old S. mutans biofilms $(p<0.05)$. Data represent mean \pm standard deviation. ${ }^{\star} p<0.05$ : significantly different from each other. 

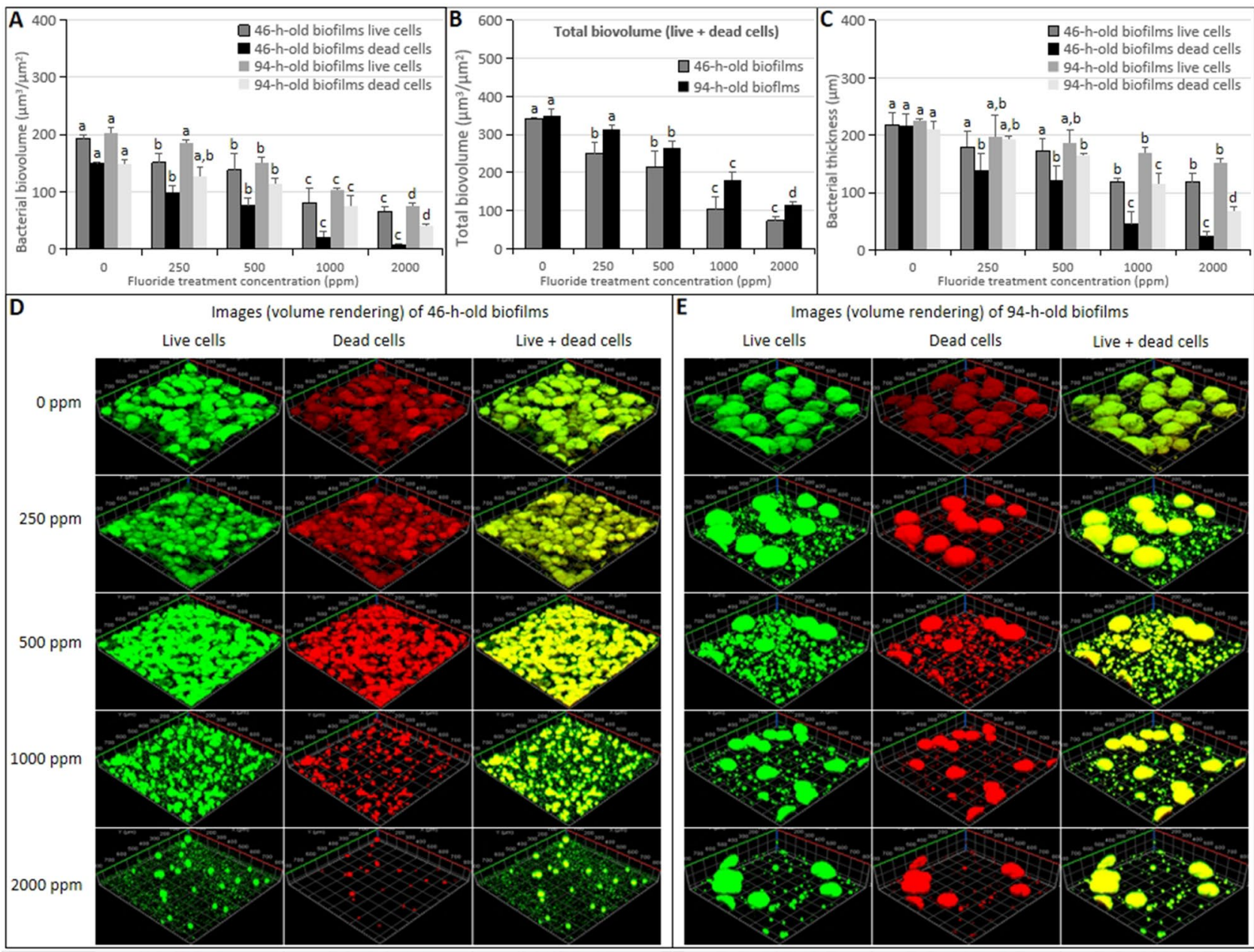

Live + dead cells

Figure 4. Change in confocal laser scanning microscopy of bacterial cells in the 46/94-h-old S. mutans biofilms treated with different concentrations of fluoride. (A) Bacterial biovolume. (B) Total biovolume (live + dead cells). (C) Bacterial thickness. (D) Representative confocal images of 46-h-old S. mutans biofilms. (E) Representative confocal images of 94 -h-old $S$. mutans biofilms. Data represent mean \pm standard deviation. Significantly different from each other $(p<0.05)$.

biofilm cells, and it showed the highest final $\mathrm{H}^{+}$concentration, indicating the most pronounced effect on the acid resistance of biofilm cells (Fig. 3A).

Confocal laser scanning microscope study of early S. mutans biofilms. To further evaluate the effect of brief fluoride treatment on early $S$. mutans biofilms formation components and structure, the confocal laser scanning microscopy analysis was performed. As shown in Fig. 4, the bacterial biovolumes of live/ dead cells, bacterial thicknesses, and total biovolumes of the 46-h-old $S$. mutans biofilms were reduced by brief fluoride treatments in a concentration-dependent manner. The lowest values were found for the $2000 \mathrm{ppm}$ fluoride treatment group (Fig. 4A-C; $p<0.05$ ). Significant differences in the bacterial biovolumes of live/dead cells, bacterial thicknesses, and total biovolumes of the 46-h-old $S$. mutans biofilms were detected between each treatment group and the control group. The three-dimensional image of bacterial microcolonies of 46-h-old S. mutans biofilms showed that with the increased concentration of the brief fluoride treatment, the morphology was changed to reflect a lower number of the live/dead cells microcolonies with smaller sizes and looser arrangements (Fig. 4D). Brief fluoride treatments also affected EPS formation of $S$. mutans biofilms, as shown in Fig. 5A,B. Brief fluoride treatments reduced EPS biovolumes $(p<0.05)$ and thicknesses $(p>0.05)$. With the increased concentration of the brief fluoride treatment, the anti-EPS formation increased in a concentrationdependent manner. EPSs and bacterial microcolony images of 46-h-old S. mutans biofilms showed minimal concentrations of homogeneous structures of EPSs covering and surrounding bacterial microcolonies for the 2000 ppm fluoride treatment group (Fig. 5C).

Effects of brief fluoride treatments during mature $S$. mutans biofilms formation. The microbiological and biochemical analyses in the mature $S$. mutans biofilms formation. As shown in Fig. 1, only the treatment group with $2000 \mathrm{ppm}$ fluoride presented significant inhibition of dry weight accumulation, bacterial 


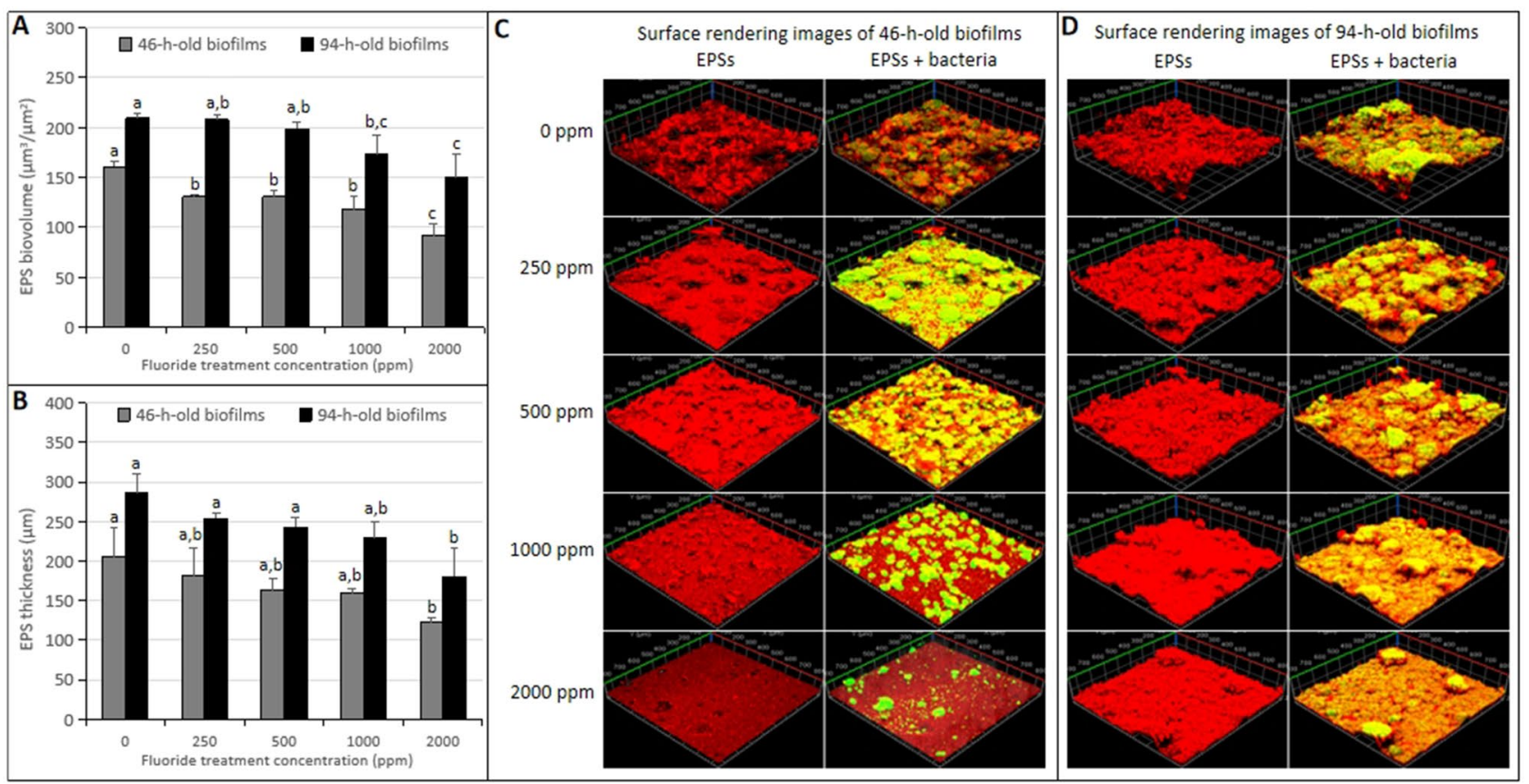

Figure 5. Change in confocal laser scanning microscopy of EPSs in the 46/94-h-old S. mutans UA159 biofilms treated with different concentrations of fluoride. (A) EPS biovolume. (B) EPS thickness. (C) Representative confocal images of 46-h-old S. mutans biofilms. (D) Representative confocal images of 94-h-old S. mutans biofilms. Data represent mean \pm standard deviation. Significantly different from each other $(p<0.05)$.

activity, and polysaccharides formation of the 94-h-old S. mutans biofilms compared with those of the control and other fluoride treatment groups (Fig. 1A-C, p >0.05; Fig. 1D,E, $\mathrm{p}>0.05$ ). The lowest values were found for the 2000 ppm fluoride treatment group.

Relationship between fluoride concentration and acid production of mature $S$. mutans biofilms. Figure $2 \mathrm{~B}$ showed the $\mathrm{pH}$ changes of the old culture medium, it was found that all tested concentrations of fluoride treatment groups inhibited the acid-producing capacity of the biofilm in the mature stage of the biofilm $(p<0.05)$. The concentration of acid in the old medium decreased in a concentration-dependent manner, and the highest inhibition ability was shown for the $2000 \mathrm{ppm}$ fluoride treatment group. As shown in Fig. 3C,D, brief fluoride treatments inhibited acid production in the mature stage of cariogenic biofilms. The acidification activities of biofilms were inhibited in all fluoride treatment groups, and the $2000 \mathrm{ppm}$ fluoride treatment group showed the lowest initial rate and total acid production (Fig. 3D). Additionally, the $2000 \mathrm{ppm}$ fluoride treatment group showed reduced acid resistance of cariogenic biofilm cells, and it showed the highest final $\mathrm{H}^{+}$concentration, indicating the most pronounced effect on the acid resistance of biofilm cells (Fig. 3C).

Confocal laser scanning microscope study of mature S. mutans biofilms. As shown in Fig. 4, the bacterial biovolumes of live/dead cells, bacterial thicknesses, and total biovolumes of 94-h-old S.mutans biofilms were reduced by brief fluoride treatments in a concentration-dependent manner. The lowest values were found for the $2000 \mathrm{ppm}$ fluoride treatment group (Fig. 4A-C; $p<0.05$ ). Significant differences in 94-h-old $S$. mutans biofilms of the treatment group and the control group were only detected for treatment by fluoride with a minimum concentration of $500 \mathrm{ppm}$. Three-dimensional images showed that the live/dead cells in the 94-hold biofilms showed larger microcolonies than those of the 46-h-old biofilms and uniform morphologies and compact arrangements. In addition, the number of live/dead cells microcolonies decreased and the arrangement of microcolonies became loose with the increased concentration of fluoride treatment, but the morphologies of microcolonies did not change significantly, and microcolonies in each treatment group were similar to those of the control group (Fig. 4E). Brief fluoride treatments also affected EPS formation of $S$. mutans biofilms, as shown in Fig. 5A,B. Brief fluoride treatment reduced EPSs biovolumes and thicknesses $(p<0.05)$. With the increased concentration of the brief fluoride treatment, the anti-EPS formation increased in a concentration-dependent manner. EPSs and bacterial microcolony images of 94-h-old S. mutans biofilms showed minimal concentrations of homogeneous structures of EPSs covering and surrounding bacterial microcolonies for the 2000 ppm fluoride treatment group (Fig. 5D).

\section{Discussion}

Multiple pieces of evidence have supported the proposal that fluoride affects acidogenicity, acid tolerance, and EPS formation of cariogenic biofilms, such as $S$. mutans biofilms ${ }^{18-20}$. However, the effects of fluoride on the biological activity of oral microorganisms and biofilm activity at different stages of cariogenic biofilm formation have rarely been studied. In the present study, the $S$. mutans biofilm model was used to explore the effects of different 
concentrations of fluoride on the growth, virulence (EPSs and acidogenicity), and activity of early and mature $S$. mutans biofilms. In fact, the $S$. mutans biofilm model is unable to precisely mimic the complex microbial community observed in dental biofilms, but monospecies biofilms are advantageous in examining the mechanisms of $S$. mutans in biofilms with sufficient performance to validate data reproducibility and reduce variance ${ }^{10,21,22}$.

Biofilms are typically characterized by dense, highly hydrated clusters of bacterial cells. Bacterial adhesion and early biofilm formation are the first steps of cariogenic biofilm formation ${ }^{23,24}$. In the early stage of biofilm formation, the biovolumes and average thicknesses of bacterial microcolonies and EPSs of biofilms continuously increase $\mathrm{e}^{25}$. At the mature biofilm stage beginning after $46 \mathrm{~h}$, the number of CFUs and acid production capacity of the biofilm remained stable, as did the bacterial microflora of the biofilm and the biovolume and average thickness of the EPSs. The standard deviation of the biovolume and mean biofilm thickness of bacteria or EPSs remained unchanged over time ${ }^{25}$. Mature biofilms have thicker and more complex biofilm structures, which may affect the diffusion and penetration of antibacterial agents ${ }^{26,27}$. Therefore, the antimicrobial membrane activity of $S$. mutans treated briefly with fluoride during biofilm formation was studied here. As shown in Fig. 1, brief fluoride treatments with all tested concentrations significantly reduced the dry weight accumulations and bacterial activities of 46-h-old $S$. mutans biofilms in a concentration-dependent manner, and the differences between each treatment group and the control group were statistically significant (Fig. $1 \mathrm{~A} ; p<0.05$ ). Notably, the activity of biofilm bacteria was significantly affected in the experimental groups treated with fluoride at a minimum concentration of $500 \mathrm{ppm}$ compared with that of the control group (Fig. 1B; $p<0.05$ ). However, only the treatment group with $2000 \mathrm{ppm}$ fluoride presented significant inhibition of dry weight accumulation and bacterial activity in the 94-h-old $S$. mutans biofilms compared with those of the control and other fluoride treatment groups (Fig. 1A,B; $p>0.05$ ).

As shown by confocal laser scanning, the bacterial biovolumes of live/dead cells, total biovolumes, and bacterial thicknesses of the 46-h-old and 94-h-old S. mutans biofilms were reduced by brief fluoride treatments in a concentration-dependent manner. The lowest values were found for the $2000 \mathrm{ppm}$ fluoride treatment group. Significant differences in the bacterial biovolumes of live/dead cells, total biovolumes, and bacterial thickness of the 46-h-old S. mutans biofilms were detected between each treatment group and the control group. However, significant differences in 94-h-old $S$. mutans biofilms of the treatment group and the control group were only detected for treatment by fluoride with a minimum concentration of $500 \mathrm{ppm}$ (Fig. 4A-C; $p<0.05$ ). The morphology of live/dead cell microcolonies of the 46-h-old $S$. mutans biofilms was uniform and tightly arranged in the control group. With the increased concentration of the brief fluoride treatment, the morphology was changed to reflect a lower number of live/dead cell microcolonies with smaller sizes and looser arrangements. High-concentration fluoride treatments at the early stage of $S$. mutans biofilms significantly affected the structural integrities of $S$. mutans biofilm cells (Fig. 4D). The live/dead cells in the 94-h-old biofilms showed larger microcolonies than those of the 46-h-old biofilms and uniform morphologies and compact arrangements. In addition, the number of live/dead cell microcolonies decreased and the arrangements of microcolonies became looser with the increased concentrations of fluoride treatment, but the morphologies of microcolonies did not change significantly, and microcolonies in each treatment group were similar to those of the control group (Fig. 4E). It was concluded that brief fluoride treatment was closely related to antibacterial biofilm activity, which was determined by the biofilm formation stage and the concentration of the fluoride treatment. Biofilms in early formation stages were more susceptible to the influence of fluoride than those in mature stages. The antibacterial biofilm activities of biofilms in the high-concentration fluoride treatment group were higher than those of the low-concentration fluoride treatment groups. According to the results of live/dead cell microcolonies, brief fluoride treatments did not have a bactericidal or killing effect on the growth of $S$. mutans biofilms. It has been reported that fluoride treatments with concentrations of 3040-5700 ppm or $>5000 \mu \mathrm{g} / \mathrm{ml}$ had bactericidal effects on S. mutans ${ }^{28,29}$. In this study, the highest concentration of a fluoride treatment was $2000 \mathrm{ppm}$, which was lower than those with reported bactericidal activity.

Polysaccharides account for up to $40 \%$ of the dry weights of dental biofilms, and they are typically synthesized by microbial glucosyltransferases ${ }^{30}$. Therefore, the reduction in biofilm biomass is directly related to the reduction of polysaccharide levels in the overall biofilm matrix. The complex structural integrity of a biofilm is mainly determined by the density of formation, volume, structural integrity, and stability, which are also associated with EPSs ${ }^{7,31}$. In this study, the effects of brief fluoride treatments on the synthesis of water-soluble/ insoluble extracellular and intracellular polysaccharides during the formation of $S$. mutans biofilms were investigated. The synthesis of water-soluble/insoluble extracellular and intracellular polysaccharides in 46-h-old $S$. mutans biofilms was significantly inhibited in all fluoride treatment groups compared with the control group. The anti-EPS formation activity increased in a concentration-dependent manner (Fig. $1 \mathrm{C}-\mathrm{E} ; p<0.05$ ). In the 94-h-old S. mutans biofilms, only the $2000 \mathrm{ppm}$ fluoride treatment group significantly inhibited the synthesis of water-insoluble extracellular and intracellular polysaccharides compared with the control group (Fig. 1C, $p>0.05$; Fig. 1E, $\mathrm{p}<0.05)$. In addition, the synthesis of water-soluble EPSs was significantly inhibited in the $1000 \mathrm{ppm}$ and 2000 ppm fluoride treatment groups compared with the control group (Fig. 1D; $p<0.05$ ).

Since the biofilms are mainly composed of bacterial cells and polysaccharides ${ }^{7}$, the decrease in dry weight in all fluoride treatment groups of the 46-h-old S. mutans biofilms and $2000 \mathrm{ppm}$ fluoride treatment group of the 94-h-old $S$. mutans biofilms may be attributed to the decreased number of bacterial cells and suppressed EPS formation. The biovolumes and thicknesses of EPSs in S. mutans biofilms detected by confocal laser scanning microscopy also supported our findings. The formation of EPSs in biofilms was remarkably damaged by brief fluoride treatment, and the decrease in biovolumes and thicknesses of EPSs may further affect the growth height of bacterial cells (Fig. 5). Brief fluoride treatments inhibited the synthesis of biofilm polysaccharides, which was closely linked with the stage of biofilm formation and the concentrations of fluoride treatments. Compared with early-stage biofilms, a higher concentration of fluoride $(\geq 1000 \mathrm{ppm})$ was required to significantly inhibit the synthesis of polysaccharides in the mature stages of cariogenic biofilms. In our study, the inhibitory capacity of 
the high concentration fluoride treatment group was higher than that of the low concentration fluoride treatment group. The inhibitory effects of brief fluoride treatments on polysaccharide syntheses in biofilms may be attributed to inhibition of glucosyltransferases activity or its production in S. mutans biofilm cells. The exact mechanism by which brief fluoride treatments inhibit glucosyltransferase activity and production in S. mutans biofilm cells, however, needs to be explored in the future.

Acid production by carbohydrates and acid tolerance in low $\mathrm{pH}$ environments are among the main toxicity characteristics of $S$. mutans $^{7}$, which are closely associated with enamel demineralization and the formation of dental caries ${ }^{32-34}$. It is well known that fluoride has inhibitory effects on the acidogenicity of cariogenic biofilms ${ }^{14}$. In this study, the acid production and acid tolerance of $S$. mutans biofilm formation by brief fluoride treatment were studied. The results showed that fluoride treatments inhibited acid production and acid tolerance in S. mutans biofilm cells (Figs. 2, 3). In the $\mathrm{pH}$ changes of the old culture medium, it was found that all tested concentrations of fluoride treatment groups inhibited the acid-producing capacity of the biofilm in the early formation and mature stages of the biofilm. The concentration of acid in the old medium decreased in a concentration-dependent manner, and the highest inhibition ability was shown for the 2000 ppm fluoride treatment group (Fig. 2A,B). The decreases in acid concentrations may be related to decreases in biofilm cell viability or physiological capacity. In the glycolytic $\mathrm{pH}$ drop experiment, the initial $\mathrm{pH}$ value reflected the acid-producing capacity of the cell, and the final $\mathrm{pH}$ value reflected the acid-tolerance capacity of the cell $^{34}$. As shown in Fig. 3, brief fluoride treatments inhibited acid production in the early and mature stages of cariogenic biofilms. The acidification activities of biofilms were inhibited in all fluoride treatment groups, and the 2000 ppm fluoride treatment group showed the lowest initial rate and total acid production (Fig. 3B,D). Additionally, the $2000 \mathrm{ppm}$ fluoride treatment group showed reduced acid resistance of cariogenic biofilm cells, and it showed the highest final $\mathrm{H}^{+}$concentration, indicating the most pronounced effect on the acid resistance of biofilm cells (Fig. 3A,C). The change in $\mathrm{H}^{+}$concentration may be caused by the inhibition of glycolytic acid production by fluoride treatment. These results indicated that acid production and acid tolerance of the biofilm could be reduced by brief fluoride treatments performed during the early stage of biofilm formation and the mature stage. Anti-acidification activity was correlated with the concentration of fluoride, and the $2000 \mathrm{ppm}$ fluoride treatment achieved the highest anti-acidification activity.

In general, brief fluoride treatments significantly inhibited the formation of $S$. mutans biofilms in the early stage. At the early stages of biofilm formation, the levels of dry weight, bacterial activity, water-soluble/insoluble polysaccharides, intracellular polysaccharides, and acid-producing capacity of $S$. mutans biofilms were significantly inhibited in all fluoride treatment groups compared with those of the control group (Figs. $1,2,3 ; p<0.05)$. The inhibited growth of cariogenic bacteria in early-stage $S$. mutans biofilms was positively correlated with the concentration of fluoride treatment. At the mature biofilm stage, only the $2000 \mathrm{ppm}$ fluoride treatment group significantly inhibited the growth of $S$. mutans biofilms compared with the control group. It is suggested that the inhibitory effects of brief fluoride treatments on the activity of $S$. mutans biofilms depend on the stage of biofilm formation. In the early stage of biofilm formation, fluoride treatment at a low concentration significantly inhibited the growth of biofilms, while a high concentration was required in the mature stage. Our experimental results also showed that the inhibitory effects of fluoride treatments on the growth of $S$. mutans biofilms either in the early or mature stage were related to the concentration of the fluoride treatment. Compared with other fluoride treatment groups and the control group, the $2000 \mathrm{ppm}$ fluoride treatment group exhibited the strongest capability of inhibiting the growth of $S$. mutans biofilms. This study provides a theoretical basis for the timing of fluoride treatments used to prevent dental caries. However, further research is needed to reveal the exact mechanism underlying the role of fluoride treatments in inhibiting early-stage cariogenic biofilm formation.

\section{Conclusion}

The antimicrobial activities of fluoride treatments on the growth of $S$. mutans biofilms were linked with the stage of biofilm formation. Brief fluoride treatments significantly inhibited the growth of early-stage $S$. mutans biofilms. The inhibition of $S$. mutans biofilm growth by fluoride treatment was easier in the early formation stage than in the mature stage. Fluoride treatment in the early stage of cariogenic biofilm formation may be an effective approach to controlling cariogenic biofilm development and preventing dental caries.

\section{Materials and methods}

Streptococcus mutans biofilms formation, fluoride, and experimental scheme. Figure 6 shows S. mutans biofilm preparation and experimental scheme for the present study. S. mutans UA159 (ATCC 700610; serotype c) biofilms were formed on saliva-coated hydroxyapatite discs $\left(2.93 \mathrm{~cm}^{2}\right.$; Clarkson Chromatography Products, Inc., South Williamsport, PA, USA) placed in a vertical position in 24-well plates. Briefly, an adult male was selected for oral saliva collection. hydroxyapatite discs were incubated in filter-sterilized $(0.22-\mu \mathrm{m}$ low protein-binding filter) saliva $\left(3 \mathrm{ml} /\right.$ disc) for $1 \mathrm{~h}$ at $37^{\circ} \mathrm{C}$. For biofilms formation, the saliva-coated hydroxyapatite discs were transferred to a 24 -well plate containing brain heart infusion (BHI; D-ifco, Detroit, MI, USA) broth with $1 \%(\mathrm{w} / \mathrm{v})$ sucrose and $S$. mutans UA159 $\left(5-7 \times 10^{6} \mathrm{CFU} / \mathrm{ml}\right)(3 \mathrm{ml} / \mathrm{disc})$. The biofilms were grown at $37^{\circ} \mathrm{C}$ with $5 \% \mathrm{CO}_{2}$. After $22 \mathrm{~h}$ of biofilm growth, the culture medium was changed twice daily ( 9 a.m. and 6 p.m.) until it was $46 \mathrm{~h}\left(0-46 \mathrm{~h}\right.$ early biofilm formation) or $94 \mathrm{~h}(46-94 \mathrm{~h} \text { mature biofilm formation })^{10,15-17}$. In the present study, the biofilms of $\geq 46 \mathrm{~h}$ were defined as mature biofilms ${ }^{25,35}$. This study is approved by the ethics committee/institutional review board of the Department of Preventive Dentistry, School of Dentistry, Institute of Oral Bioscience, Jeonbuk National University. All experimental protocols were approved by the Department of Preventive Dentistry, School of Dentistry, Institute of Oral Bioscience, Jeonbuk National University. The author confirms that all methods were carried out in accordance with relevant guidelines and regulations. 


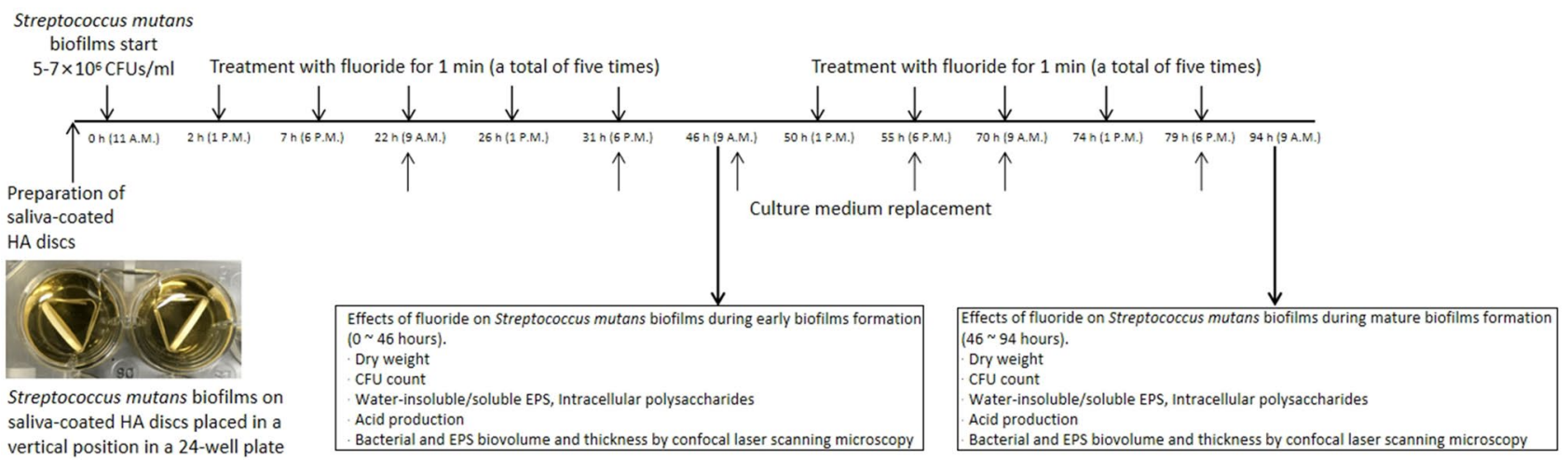

Figure 6. Streptococcus mutans biofilms formation and experimental scheme.

The fluoride source in this study was $\mathrm{NaF}$. NaF was purchased from Sigma-Aldrich (St Louis, MO, USA). The solutions were made with $\mathrm{NaF}$ and purified water. Fluoride products at concentrations between 1 to $2000 \mathrm{ppm}$ are recommended ${ }^{8}$. In this study, four concentrations of fluoride were used for the experiment, respectively: $250 \mathrm{ppm}, 500 \mathrm{ppm}, 1000 \mathrm{ppm}, 2000 \mathrm{ppm}$.

To determine the anti-cariogenic biofilm activity of brief fluoride treatments during early $S$. mutans biofilm formation, the saliva-coated hydroxyapatite disks were treated with $0 \mathrm{ppm}$ (control group), $250 \mathrm{ppm}, 500 \mathrm{ppm}$, $1000 \mathrm{ppm}, 2000 \mathrm{ppm}$ for $1 \mathrm{~min}$, a total of 5 times treated during the early S. mutans biofilm formation (at 2, 7, $22,26,31 \mathrm{~h}$ ). The treated saliva-coated hydroxyapatite disks were washed with distilled water three times, then transferred into the original 24 -well plates containing a $1 \%$ sucrose culture medium. The incubated time of the S. mutans biofilms was $46 \mathrm{~h}$.

To determine the anti-cariogenic biofilm activity of brief fluoride treatments during mature $S$. mutans biofilms formation, $S$. mutans biofilms were not treated at the early biofilm growth stage. After mature biofilm formation, the saliva-coated hydroxyapatite disks were treated with $0,250,500,1000,2000 \mathrm{ppm}$ for $1 \mathrm{~min}$, a total of 5 times treated during the mature $S$. mutans biofilms formation (at 50, 55, 70, 74, $79 \mathrm{~h}$ ). The treated saliva-coated hydroxyapatite disks were washed with distilled water three times, then transferred into the original 24-well plates containing a $1 \%$ sucrose culture medium. The incubated time of the $S$. mutans biofilms was $94 \mathrm{~h}$.

The effects of different concentrations of fluoride on the dry weight, CFUs, water-soluble/insoluble EPSs, intracellular polysaccharide, and acidogenicity of early and mature biofilms were compared. The images of biofilms at 46-h-old and 94-h-old were obtained by confocal laser scanning microscopy.

Microbiological and biochemical biofilm analyses. The dry weight and CFUs in the homogenized suspension were analyzed. Briefly, the 46/94-h-old biofilms on the saliva-coated hydroxyapatite disc were transferred into $2 \mathrm{ml}$ of $0.89 \% \mathrm{NaCl}$ and sonicated in an ultrasonic bath (Power sonic 410; Hwashin Technology Co., Seoul, Korea) for $10 \mathrm{~min}$ to disperse the biofilms. The dispersed solution was re-sonicated at $7 \mathrm{~W}$ for $30 \mathrm{~s}$ after adding $3 \mathrm{ml}$ of $0.89 \% \mathrm{NaCl}$ (VCX $130 \mathrm{~PB}$; Sonics and Materials, Inc., Newtown, CT, USA). For the determination of CFUs count, an aliquot $(0.1 \mathrm{ml})$ of the homogenized solution $(5 \mathrm{ml})$ was serially diluted, plated onto brain heart infusion (BHI; Difco, Detroit, MI, USA) agar plates, and then incubated under aerobic conditions at $37^{\circ} \mathrm{C}$ to determine the CFUs count ${ }^{15,36}$.

For the determination of the dry weight and amount of water-insoluble EPSs, water-soluble EPSs, intracellular polysaccharides, the remaining solution $(4.9 \mathrm{ml})$ was centrifuged $(3000 \times g)$ for $20 \mathrm{~min}$ at $4{ }^{\circ} \mathrm{C}$. The biofilm pellet was resuspended and washed twice in the same volume of water. Mix the water washed the biofilms pellet with $95 \%$ alcohol and put it in a refrigerator at $-20^{\circ} \mathrm{C}$ for at least $18 \mathrm{~h}$ to precipitate the water-soluble EPSs. Then calculated the content of water-soluble EPSs in the biofilms. The washed biofilms pellet is evenly divided into two portions, lyophilized, and weighed to determine the dry weight. One part used $1 \mathrm{~N}$ sodium hydroxide to extract water-insoluble EPSs from the dried precipitate. The other part was used to calculate the content of intracellular polysaccharides, as detailed elsewhere ${ }^{21}$.

Acid production analysis. The final $\mathrm{pH}$ values of the old culture media were also determined during the experimental period using a glass electrode (Beckman Coulter Inc., Brea, CA, USA) to investigate the change in acidogenicity of $S$. mutans biofilms by the treatments. The effect of brief fluoride treatment on the acidogenic and aciduric activity of early and mature $S$. mutans biofilms was determined by the glycolytic $\mathrm{pH}$ drop assay. Briefly, $S$. mutans biofilms were not treated with fluoride during the formation stage. the 46/94-h-old $S$. mutans biofilm was incubated in $20 \mathrm{mM}$ potassium phosphate buffer ( $\mathrm{pH}$ 7.2) for $1 \mathrm{~h}$ to deplete endogenous catabolites. They were then washed with salt solution $\left(50 \mathrm{mM} \mathrm{KCl}+1 \mathrm{mM} \mathrm{MgCl}_{2}, \mathrm{pH} 7.0\right)$ and treated with fluoride $(0,250,500,1000,2000 \mathrm{ppm} \mathrm{F}-)$. After the fluoride treatment, the biofilms were dip-washed with salt solution and transferred into a 6-well plate containing salt solution. The $\mathrm{pH}$ was adjusted to 7.2 with a $0.2 \mathrm{M} \mathrm{KOH}$ solution. Glucose was then added to the mixture to give a final concentration of $1 \%(\mathrm{w} / \mathrm{v})$. The decrease in $\mathrm{pH}$ was assessed using a glass electrode over $120 \mathrm{~min}$ (Futura Micro Combination $\mathrm{pH}$ electrode, $5 \mathrm{~mm}$ diameter; Beckman Coulter Inc., CA, USA). The effect of fluoride on the acid production of the biofilm was determined according to the acid production rate, calculated by the change in $\mathrm{pH}$ values over the linear portion $(0-20,30$, $120 \mathrm{~min}$ ) of the $\mathrm{pH}$ drop curves ${ }^{37}$. 
The initial rate (0-20 min) of $\mathrm{H}^{+}$production (y1) and initial rate $(0-30 \mathrm{~min})$ of $\mathrm{H}^{+}$production $(\mathrm{y} 2)$ was derived from the equation:

$$
\begin{aligned}
& \mathrm{y} 1=\left(\mathrm{H}^{+} \text {concentration at } 20 \mathrm{~min}-\mathrm{H}^{+} \text {concentration at } 0 \mathrm{~min}\right) / 20 . \\
& \mathrm{y} 2=\left(\mathrm{H}^{+} \text {concentration at } 30 \mathrm{~min}-\mathrm{H}^{+} \text {concentration at } 0 \mathrm{~min}\right) / 30 .
\end{aligned}
$$

The total produced concentration of $\mathrm{H}^{+}(\mathrm{y} 3)$ was derived from the equation:

$$
\mathrm{y} 3=\mathrm{H}^{+} \text {concentration at } 120 \mathrm{~min}-\mathrm{H}^{+} \text {concentration at } 0 \mathrm{~min} \text {. }
$$

Confocal laser scanning microscopy analysis. Live and dead bacterial cells staining. Confocal laser scanning microscopy analysis was performed to confirm the results of microbiological and biochemical studies. To investigate the difference in bacterial cells, the 46/94-h-old biofilms were stained at room temperature in the dark for 30 min using the Film Tracer LIVE/DEAD Biofilm viability kit L10316 (Invitrogen, Molecular Probes Inc., Eugene, OR, USA). The final concentrations of SYTO 9 and propidium iodide (PI) were 6.0 and $30 \mu \mathrm{M}$, respectively. This viability kit was based on plasma membrane integrity to determine live and dead cells. In this study, we regarded the cells with intact membranes (green) as live cells, whereas cells with damaged membranes (red) were regarded as dead cells. The excitation/emission wavelengths were $480 / 500 \mathrm{~nm}$ for SYTO 9 and 490/635 nm for PI for collecting the fluorescence. The stained live and dead bacterial cells were observed with an LSM 510 META microscope (Carl Zeiss, Jena, Germany) equipped with argon-ion and helium-neon lasers. All confocal fluorescence images were taken with an EC Plan-Neofuar 10x/0.30 M27 objective lens. A stack of slices in $6.4 \mu \mathrm{m}$ step sizes was captured from the top to the bottom of the biofilms. The biovolume and thickness of live and dead cells were quantified from the entire stack using COMSTAT image-processing software. The biovolume is defined as the volume of the biomass $\left(\mu \mathrm{m}^{3}\right)$ divided by the substratum (hydroxyapatite surface) area $\left(\mu \mathrm{m}^{2}\right)$. The three-dimensional architecture of the biofilms was visualized using ZEN 2.3 (blue edition) (Carl Zeiss Microscopy GmbH, Jena, Germany). The original confocal data was uploaded to ZEN 2.3 software and the intensity of green and red fluorescence in the full thickness of biofilms layers were captured automatically. The software reconstructed the 2-dimensional intensity of fluorescence in all the layers to a 3-dimensional volume stack ${ }^{38}$.

EPS staining. The EPSs of 46/94-h-old biofilms were also investigated by simultaneous in situ labeling as described elsewhere ${ }^{39}$. Briefly, Alexa Fluor ${ }^{\circledR} 647$-labeled dextran conjugate $(1 \mu \mathrm{M}, 10,000 \mathrm{MW}$; absorbance/fluorescence emission maxima 647/668 nm; Molecular Probes Inc., Eugene, OR, USA) was added to the culture medium during the formation of $S$. mutans biofilms (at 0, 22, 31 h of 46-h-old biofilms; at 0, 22, 31, 46, 55, 70, $79 \mathrm{~h}$ of 94 -h-old biofilms) to label the newly formed EPSs. As described above, the stained EPSs were observed with an LSM 510 META microscope (Carl Zeiss, Jena, Germany) (objective: EC Plan Neofuar 10x/0.30 M27) equipped with argon-ion and helium-neon lasers and visualized using ZEN 2.3. A stack of slices in $7.8 \mu \mathrm{m}$ step sizes was captured from the top to the bottom of the biofilms. Four independent experiments were performed, and five image stacks per experiment were collected. The EPSs biovolume and thickness were quantified from the confocal stacks using COMSTAT.

Statistical analysis. All experiments (except CLSM and SEM) were performed in duplicate, and at least six different experiments were conducted. The data are presented as mean \pm standard deviation. Inter-group differences were estimated using a one-way analysis of variance, followed by a post hoc multiple comparison (Tukey) test to compare multiple means (SPSS software, IBM). Values were considered statistically significant when the $p$-value was $<0.05$.

Received: 3 June 2021; Accepted: 31 August 2021

Published online: 14 September 2021

\section{References}

1. Marsh, P. D. Are dental diseases examples of ecological catastrophes?. Microbiology 149, 279-294 (2003).

2. Marsh, P. D. Controlling the oral biofilm with antimicrobials. J. Dent. 38, 11-15 (2010).

3. Takahashi, N. \& Nyvad, B. Caries ecology revisited: Microbial dynamics and the caries process. Caries Res. 42, 409-418 (2008).

4. Beighton, D. The complex oral microflora of high-risk individuals and groups and its role in the caries process. Community Dent. Oral Epidemiol. 33, 248-255 (2005).

5. Hojo, K., Nagaoka, S., Ohshima, T. \& Maeda, N. Bacterial interactions in dental biofilm development. J. Dent. Res. 88, 982-990 (2009).

6. Lemos, J. A., Quivey, R. G., Koo, H. \& Abranches, J. Streptococcus mutans: A new Gram-positive paradigm?. Microbiology 159, 436-445 (2013).

7. Bowen, W. H. \& Koo, H. Biology of Streptococcus mutans-derived glucosyltransferases: Role in extracellular matrix formation of cariogenic biofilms. Caries Res. 45, 69-86 (2011).

8. Centers for Disease Control and Prevention. Recommendations for using fluoride to prevent and control dental caries in the United States. MMWR Morb. Mortal. Wkly. Rep. 50, 1-42 (2001).

9. ten Cate, J. M. Current concepts on the theories of the mechanism of action of fluoride. Acta Odontol. Scand. 57, 325-329 (1999).

10. Pandit, S., Kim, J. E., Jung, K. H., Chang, K. W. \& Jeon, J. G. Effect of sodium fluoride on the virulence factors and composition of Streptococcus mutans biofilms. Arch. Oral Biol. 56, 643-649 (2011).

11. Loskill, P. et al. Reduced adhesion of oral bacteria on hydroxyapatite by fluoride treatment. Langmuir 29, 5528-5533 (2013).

12. Bowen, W. H. \& Hewitt, M. J. Effect of fluoride on extracellular polysaccharide production by Streptococcus mutans. J. Dent. Res. 53, 627-629 (1974). 
13. Marquis, R. E., Clock, S. A. \& Mota-Meira, M. Fluoride and organic weak acids as modulators of microbial physiology. FEMS Microbiol. Rev. 760, 1-18 (2003).

14. Pandit, S., Kim, H. J., Song, K. Y. \& Jeon, J. G. Relationship between fluoride concentration and activity against virulence factors and viability of a cariogenic biofilm: In vitro study. Caries Res. 47, 539-547 (2013).

15. Pandit, S., Cai, J. N., Jung, J. E. \& Jeon, J. G. Effect of 1-minute fluoride treatment on potential virulence and viability of a cariogenic biofilm. Caries Res. 49, 449-457 (2015).

16. Dang, M. H., Jung, J. E., Lee, D. W., Song, K. Y. \& Jeon, J. G. Recovery of acid production in Streptococcus mutans biofilms after short-term fluoride treatment. Caries Res. 50, 363-371 (2016).

17. Pandit, S., Jung, J. E., Choi, H. M. \& Jeon, J. G. Effect of brief periodic fluoride treatments on the virulence and composition of a cariogenic biofilm. Biofouling 34, 53-61 (2018).

18. Bradshaw, D. J., Marsh, P. D., Hodgson, R. J. \& Visser, J. M. Effects of glucose and fluoride on competition and metabolism within in vitro dental bacterial communities and biofilms. Caries Res. 36, 81-86 (2002).

19. Pandit, S., Kim, H. J., Park, S. H. \& Jeon, J. G. Enhancement of fluoride activity against Streptococcus mutans biofilms by a substance separated from Polygonum cuspidatum. Biofouling 28, 279-287 (2012).

20. Neilands, J., Troedsson, U., Sjödin, T. \& Davies, J. R. The effect of delmopinol and fluoride on acid adaptation and acid production in dental plaque biofilms. Arch. Oral Biol. 59, 318-323 (2014).

21. Lemos, J. A., Abranches, J., Koo, H., Marquis, R. E. \& Burne, R. A. Protocols to study the physiology of oral biofilms. Methods Mol. Biol. 666, 87-102 (2010).

22. Klein, M. I. et al. Dynamics of Streptococcus mutans transcriptome in response to starch and sucrose during biofilm development. PLOS ONE 5, e13478 (2010).

23. Song, J., Choi, B., Jin, E. J., Yoon, Y. \& Choi, K. H. Curcumin suppresses Streptococcus mutans adherence to human tooth surfaces and extracellular matrix proteins. Eur. J. Clin. Microbiol. 31, 1347-1352 (2012).

24. Ronn, S. et al. Bacterial flagella explore microscale hummocks and hollows to increase adhesin. PNAS 110, 5624-5629 (2013).

25. Chul, O., Pandit, S. \& Jeon, J. G. Changes in the composition of artificial cariogenic biofilms over time. J. Korean Acad. Oral Health. 43, 118-123 (2019).

26. Robinson, C. et al. Plaque biofilms: The effect of chemical environment on natural human plaque biofilm architecture. Arch. Oral Biol. 51, 1006-1014 (2006).

27. Filoche, S., Wong, L. \& Sissons, C. H. Oral biofilms: Emerging concepts in microbial ecology. J. Dent. Res. 89, 8-18 (2010).

28. Bowden, H. W. Effects of fluoride on the microbial ecology of dental plaque. J. Dent. Res. 69, 653-659 (1990).

29. Cai, J. N. et al. Effects of combined oleic acid and fluoride at sub-MIC levels on EPS formation and viability of Streptococcus mutans UA159 biofilms. Biofouling 31, 555-563 (2015).

30. Koo, H., Xiao, J., Klein, M. I. \& Jeon, J. G. Exopolysaccharides produced by Streptococcus mutans glucosyltransferases modulate the establishment of microcolonies within multispecies biofilms. J. Bacteriol. 192, 3024-3032 (2010).

31. Koo, H. \& Jeon, J. G. Naturally occurring molecules as alternative therapeutic agents against cariogenic biofilms. Adv. Dent. Res. 21, 63-68 (2009).

32. Kwon, Y. R. et al. Bioactivity-guided separation of anti-acidogenic substances against Streptococcus mutans UA 159 from Polygonum cuspidatum. Oral Dis. 16, 204-209 (2010).

33. Zhou, L. et al. The in vitro study of ursolic acid and oleanolic acid inhibiting cariogenic microorganisms as well as biofilm. Oral Dis. 19, 494-500 (2013).

34. Gregoire, S., Singh, A. P., Vorsa, N. \& Koo, H. Influence of cranberry phenolics on glucan synthesis by glucosyltransferases and Streptococcus mutans acidogenicity. J. Appl. Microbiol. 103, 1960-1968 (2007).

35. Lee, D. W. et al. The antibacterial activity of chlorhexidine digluconate against Streptococcus mutans bioflms follows sigmoidal patterns. Eur. J. Oral Sci. 124, 440-446 (2016).

36. Koo, H. et al. Apigenin and tt-farnesol with fluoride effects on S. mutans biofilms and dental caries. J. Dent. Res. 84, 1016-1020 (2005).

37. Belli, W., Buckley, D. \& Marquis, R. E. Weak acid effects and fluoride inhibition of glycolysis by Streptococcus mutans GS-5. Can. J. Microbiol. 41, 785-791 (1995).

38. Zhang, T., Wang, Z., Hancock, R. E. W., Fuente-Núñez, C. D. L. \& Haapasalo, M. Treatment of oral bioflms by a D-enantiomeric peptide. PLoS ONE 23, e0166997 (2016).

39. Hwang, G. et al. Simultaneous spatiotemporal mapping of in situ $\mathrm{pH}$ and bacterial activity within an intact 3D microcolony structure. Sci. Rep. 6, 32841 (2016).

\section{Author contributions}

Y.H. designed this study, performed the experiments, analyzed the data, and wrote the main manuscript text. The author shave reviewed and approved the complete manuscript for submission.

\section{Funding}

Funding was provided by National Research Foundation of Korean grant (NRF) funded by the Korean government (MSIT) (Grant No. 2019R1H1A2080006).

\section{Competing interests}

The author declares no competing interests.

\section{Additional information}

Correspondence and requests for materials should be addressed to Y.H.

Reprints and permissions information is available at www.nature.com/reprints.

Publisher's note Springer Nature remains neutral with regard to jurisdictional claims in published maps and institutional affiliations. 
(c) (i) Open Access This article is licensed under a Creative Commons Attribution 4.0 International cc) License, which permits use, sharing, adaptation, distribution and reproduction in any medium or format, as long as you give appropriate credit to the original author(s) and the source, provide a link to the Creative Commons licence, and indicate if changes were made. The images or other third party material in this article are included in the article's Creative Commons licence, unless indicated otherwise in a credit line to the material. If material is not included in the article's Creative Commons licence and your intended use is not permitted by statutory regulation or exceeds the permitted use, you will need to obtain permission directly from the copyright holder. To view a copy of this licence, visit http://creativecommons.org/licenses/by/4.0/.

(C) The Author(s) 2021 